\title{
Modeling the Dynamics of Rabies Transmission with Vaccination and Stability Analysis
}

\author{
Tesfaye Tadesse Ega ${ }^{1, *}$, Livingstone S. Luboobi ${ }^{1,2}$, Dmitry Kuznetsov ${ }^{1}$ \\ ${ }^{1}$ CoCSE School, Nelson Mandela African Institution of Science and Technology, Arusha, Tanzania \\ ${ }^{2}$ Department of Mathematics, Makerere University, Kampala, Uganda
}

Email address:

egat@nm-aist.ac.tz (T. T. Ega), luboobi@cns.mak.ac.ug (L. S. Luboobi), Dmitry.kuznetzov@nm-aist.ac.tz (D. Kuznetsov)

\section{To cite this article:}

Tesfaye Tadesse Ega, Livingstone S. Luboobi, Dmitry Kuznetsov. Modeling the Dynamics of Rabies Transmission with Vaccination and Stability Analysis. Applied and Computational Mathematics. Vol. 4, No. 6, 2015, pp. 409-419. doi: 10.11648/j.acm.20150406.13

\begin{abstract}
In this paper we formulate a deterministic mathematical model for the transmission dynamics of rabies in human and animal within and around Addis Ababa, Ethiopia. Our model involves vaccination program for dog population. The basic reproduction number and effective reproduction numbers are computed and the results are entirely depending on the parameters of dog population, which shows the responsibility of dog population for human and livestock infection. For a specified set of values of parameters as deduced from the data provided by Ethiopian Public Health Institute of Addis Ababa, the basic reproduction number $R_{0}$ and the effective reproduction number $R_{e}$ works out to be 2 and 1.6 respectively, which indicates the disease will be endemic. The numerical simulation of reproduction ratio shows that the combination of vaccination, culling of stray dogs and controlling annual crop of new born puppies are the best method to control rabies transmission within and around Adds Ababa. The disease - free equilibrium $\varepsilon_{0}$ is computed. When the effective reproduction number $R_{e}<1$ it is proved to be globally asymptotically stable in the feasible region $\Phi$. When $R_{e}>1$ there exists one endemic equilibrium point which is locally asymptotically stable.
\end{abstract}

Keywords: Rabies, Addis Ababa, Endemic, Reproduction Number, Equilibrium Points

\section{Introduction}

Rabies is a zoonotic viral disease that causes an acute inflammation of brain in human and other warm blooded animals. It is transmitted by saliva of infected animal via bites or scratch. Once the virus enters to the body thorough skin opening, it travels by nerve tissues and the brain is a place where the virus duplicates itself. During this time the host experiences from initial symptoms like fever to hallucinations, paralysis, and eventually death [1, 17]. Salivary glands are attacked after the virus duplicate in the brain, then the saliva become the main instrument to infect other animals.

Rabies occurs in more than 150 countries and territories around the world, and it is very high in developing worlds like Africa and Asia. Poor rural communities are highly vulnerable to rabies due to interaction with domestic animals like dogs. Globally 55, 000 people die due to rabies per annum. The figure is estimated to be more due to lack of enough surveillance and under reporting in developing countries. More than $40 \%$ of the people who are bitten by infected (rabid) animals are children, which are explained by the higher tendency of children who play with animals. More than 15 million people receive post exposure vaccination worldwide to protect hundreds of thousands of exposed people $[9,16]$.

Beside its effect on human directly, rabies also has significant burden on the livestock population in Africa and Asia. This can be directly reflected by its economic effect on rabies endemic areas. Africa and Asia lose US\$ 12.3 million annually because of death of livestock due to rabies [14].

Domestic dogs are highly affected by rabies and they are the source for almost all types of human and livestock infection [6]. Classical rabies virus is mainly found in dogs worldwide and more than 3 billion people in developing countries are exposed to dog rabies. In India for example about 15 million people are bitten by dogs annually [11].

Rabies cases are reported from all regions of Ethiopia, and it has persisted for century [14]. Addis Ababa and its surroundings are the endemic parts of the country [3]. More than 2000 people are bitten by dog annually in and around Addis Ababa. A retrospective record review from 2001 to 
2009 shows that 386 deaths by rabies in human was reported with annual range of 35 to 58 . From this $42.72 \%$ were children under the age of 14 [6]. Livestock are also highly affected by rabies due to dog bites within and around Addis Ababa [14].

Mathematics has played a very important role in understanding and controlling the spread of infectious diseases, and it is a powerful tool for analyzing and predicting the dynamics of phenomena. It also helps medical professionals to organize their thinking $[7,12]$.

Some studies have been done using mathematical model to analyze the dynamics of rabies transmission among dogs and from dogs to human, but none of them incorporate livestock population which are the livelihood of poor communities.

Addo [1] developed an SEIR (Susceptible - Exposed Infected - Removed) standard model to determine and predict the spread of rabies among dogs in Bongo District Ghana. Both SEIR models with vaccination and SEIR model without vaccination were formulated with ordinary differential equations. The reproductive ratio without vaccination was determined to be greater than one which shows the virus will be endemic, and less than one with vaccination, which shows that the disease dies out. The study also applied sensitivity analysis to the model by using different number of infectious dogs and the number of dogs which had been vaccinated. The study determines the reproductive number, $R_{0}$ of rabies transmission decrease as vaccination introduce to the model. In addition to that, the model showed that rabies transmission can be decreased in the strategy of keeping dogs to the confinement place of their household.

Zhang et al. [18] developed an SEIR deterministic model to analyze and control the transmission of rabies among dogs and from dogs to human in China. Both dogs and human were included and classified into susceptible, exposed, infectious, and recovered classes. They first simulated human rabies from 1996 to 2010 using the data reported by Chinese Ministry of Health, and the numerical simulation they got significantly supported the data. They also estimate the basic reproductive rate $R_{0} \approx 2$ for rabies transmission in China. Sensitivity analysis of $R_{0}$ was performed in terms of the model parameters and compare the effects of culling and immunization of dogs. Their result shows that reducing dog birth rate and increasing dog immunization coverage rate are the most effective methods in controlling human rabies infection in china. And they recommended that culling of dogs can be replaced by immunization of dogs. The reason is that in the process of culling of dogs community can be disturbed and immigration of infected dogs can be increased. Their model predicted that rabies transmission in China will decrease for the coming 7 to 8 years and it will reach again to the peak in 2030 .

Hou et al. [8] formulated SEIV (Susceptible - ExposedInfectious-Vaccinated) model for transmission of rabies between dogs and from dogs to human in the context of Guangdong province of China. In their model both domestic and stray dogs were taken as different groups, and the model was governed by twelve differential equations. In their study the sensitivity analysis of the reproduction rate was determine in terms of various parameters. The reproduction rate was $R_{0}=1.65$ which is less compared to [18]. According to the result they found that the recruitment rate of domestic dogs, the number of stray dogs and the valid time of immunity play a very important role for the transmission of rabies.

In this study we propose a mathematical model for the transmission dynamics of rabies from dogs to both human and livestock in the context of Addis Ababa and its surrounding areas. The model is based on SEIR type and domestic dogs infect both human and livestock population. We have used improved model of Zhang et al. [18] to incorporate livestock population. Though livestock populations are highly affected by rabies virus, they have not been considered in the above studies.

\section{Materials and Method}

\subsection{Model Formulation}

In this paper we formulate SEIR (Susceptible - Exposed Infected - Recovered) model of rabies for human, dog and livestock population. We categorize each of human, dog and livestock population into susceptible, exposed, infected and recovered groups. Susceptible groups have no disease, but they are likely to be infected in case of contact with rabid dogs, Exposed individuals are those who contracted the virus via bites or scratch, but still they have not shown symptoms. Infected individuals are those who develop clinical symptoms and they are unlikely to recover due to the nature of rabies. The Recovered classes are those who recovered through vaccination before they reach to infectious stage, whereas the rest get infected and die eventually.

The human population is grouped into Susceptible, $S_{h}$, Exposed, $E_{h}$, Infectious, $I_{h}$, and Recovered, $R_{h}$, Individuals are recruited to susceptible class by birth at, $\vartheta_{h}$ to susceptible human. A susceptible man bitten by a rabid dog becomes exposed. If post exposer treatment is not given the person become infectious and end up with death since there is no recovery at infectious stage.

The dog population is divided in to Susceptible, $S_{d}$, Exposed, $E_{d}$, Infectious, $I_{d}$ and Recovered, $R_{d}$. Individuals are recruited into susceptible class by birth at $\vartheta_{d}$. For susceptible dog vaccination is applied at a rate of $\varphi_{d}$. This is because it is the dog population which infects both human and livestock. An exposed dog moves to infectious class directly. This is because the community can't observe which dog is infected since many dogs are very mobile around the city.

Livestock population also divided in to Susceptible, $S_{l}$, Exposed, $E_{l}$, Infectious, $I_{l}$ and Recovered, $R_{l}$. Individuals are recruited to susceptible class by birth at $\vartheta_{l}$. The susceptible class contracts the disease from dog bite or scratch. A livestock which is bitten by a rabid dog become exposed. If post exposure treatment is provided it moves to recovered class before reaches to infectious stage. 
Our model is developed based on the following assumptions. Susceptible populations are recruited by birth at $\vartheta$. Rabies transmission among human, livestock and each other was ignored because of negligible cases. An individual who is bitten by rabid dog become infected.

All the parameters of the model are positive and they are introduced in Table 1.

Table 1. Description of parameters.

\begin{tabular}{ll}
\hline Parameter & Description \\
\hline$\vartheta_{h}, \vartheta_{d}, \vartheta_{l}$ & $\begin{array}{l}\text { The annual birth of human, dog and livestock population } \\
\text { respectively per annum. }\end{array}$ \\
$\sigma_{h}, \sigma_{d}, \sigma_{l}$ & $\begin{array}{l}\text { Death rate due to rabies for human dog and livestock } \\
\text { population respectively. }\end{array}$ \\
$\omega_{h}, \omega_{d}, \omega_{l}$ & $\begin{array}{l}\text { The loss rate of vaccination immunity for human, dog } \\
\text { and livestock population respectively }\end{array}$ \\
$\mu_{h}, \mu_{d}, \mu_{l}$ & $\begin{array}{l}\text { Natural death rate of human, dog and livestock } \\
\text { population respectively }\end{array}$ \\
$\beta_{h}, \beta_{d}, \beta_{l}$ & $\begin{array}{l}\text { The rate of infectious dogs infects susceptible human, } \\
\text { dog and livestock respectively. }\end{array}$ \\
$\rho_{h}, \rho_{d}, \rho_{l}$ & $\begin{array}{l}\text { The incubation period in human, dog and livestock } \\
\text { population respectively. } \\
\text { Vaccination rate of exposed human, dog and livestock } \\
\text { population respectively. }\end{array}$ \\
$\varphi_{h}, \varphi_{d}, \varphi_{l}$ &
\end{tabular}

Individuals in each compartment have equal natural death rate. Populations are homogeneous, that is each individual has equal probability of dog bite and contracting the diseases. Once an individual reaches to infectious stage there is no recovery and death is $100 \%$.

Using the above assumptions, definition of variables and parameters, the model flow diagram which depicts the dynamics of rabies transmission among dogs and from dogs to both human and livestock is shown in Figure 1.

\subsection{Model Compartment and Dynamics}

The parameters of the model are positive. $\vartheta_{i}$ where $i=h, d, l$ represents the annual birth of dog, human and livestock population respectively. Exposed population of human and livestock can recover through post exposure treatment. The parameters $\rho_{i}$ where $i=h, d, l$ represent the latency rates of human, $\operatorname{dog}$ and livestock population respectively so that $\frac{1}{\rho_{i}}$ where $i=h, d, l$ are the incubation periods.

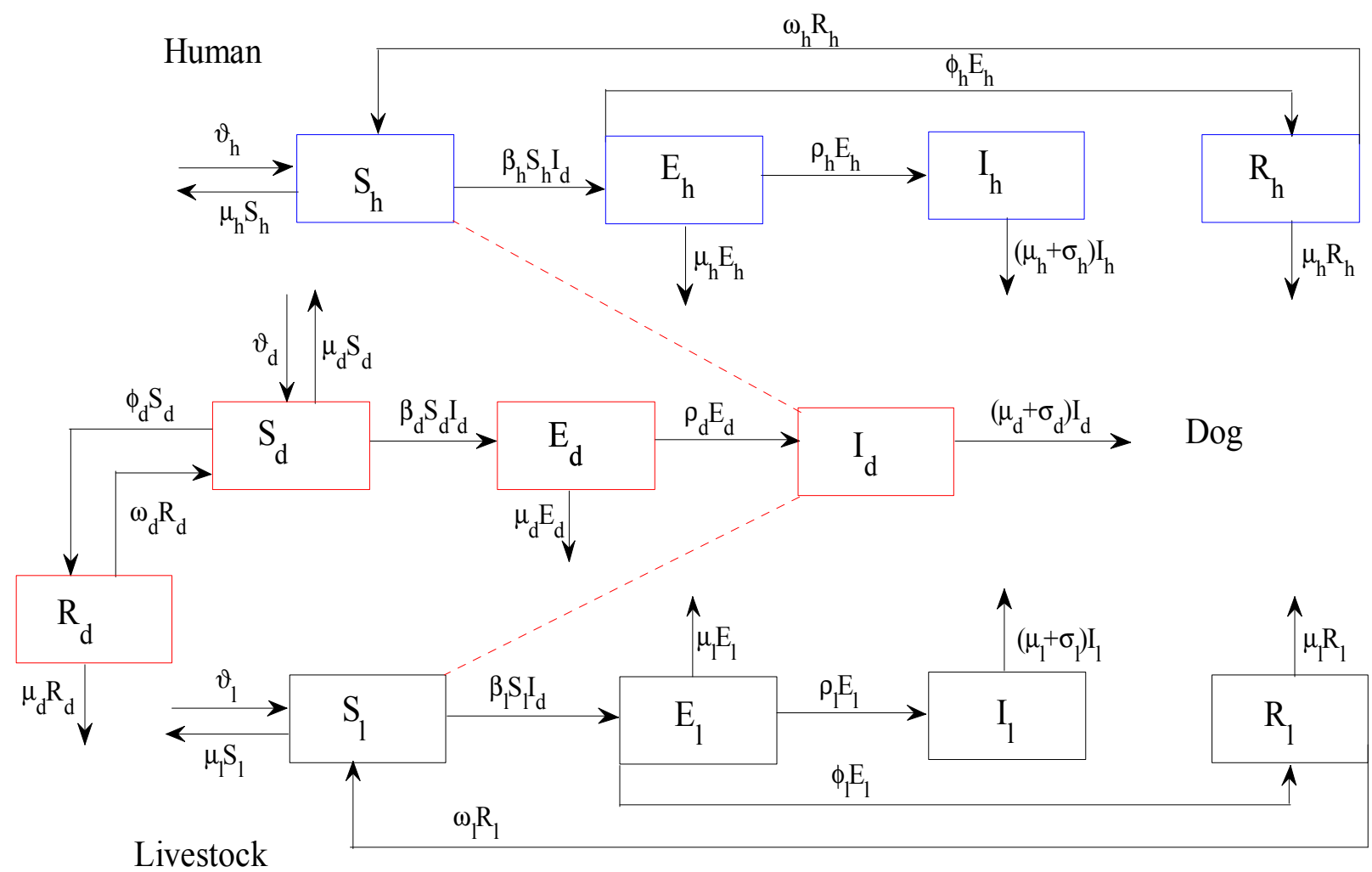

Figure 1. Flow diagram for rabies transmission among dogs and to human and livestock in which the parameters are as defined in Table 1.

\subsection{Model Equations}

Based on the assumptions and interrelation between the variables and parameters in Figure 1, rabies transmission dynamics can be described by using ordinary differential equations.

$$
\begin{aligned}
& \frac{d S_{h}}{d t}=\vartheta_{h}+\omega_{h} R_{h}-\beta_{h} I_{d} S_{h}-\mu_{h} S_{h} \\
& \frac{d E_{h}}{d t}=\beta_{h} I_{d} S_{h}-\left(\rho_{h}+\mu_{h}+\varphi_{h}\right) E_{h} \\
& \frac{d I_{h}}{d t}=\rho_{h} E_{h}-\left(\mu_{h}+\sigma_{h}\right) I_{h} \\
& \frac{d R_{h}}{d t}=\varphi_{h} E_{h}-\left(\omega_{h}+\mu_{h}\right) R_{h}
\end{aligned}
$$




$$
\begin{aligned}
& \frac{d S_{d}}{d t}=\vartheta_{d}+\omega_{d} R_{d}-\left(\mu_{d}+\varphi_{d}+\beta_{d} I_{d}\right) S_{d} \\
& \frac{d E_{d}}{d t}=\beta_{d} S_{d} I_{d}-\left(\rho_{d}+\mu_{d}\right) E_{d} \\
& \frac{d I_{d}}{d t}=\rho_{d} E_{d}-\left(\mu_{d}+\sigma_{d}\right) I_{d} \\
& \frac{d R_{d}}{d t}=\varphi_{d} S_{d}-\left(\mu_{d}+\omega_{d}\right) R_{d} \\
& \frac{d S_{l}}{d t}=\vartheta_{l}+\omega_{l} R_{l}-\beta_{l} I_{d} S_{l}-\mu_{l} S_{l} \\
& \frac{d E_{l}}{d t}=\beta_{l} I_{d} S_{l}-\left(\rho_{l}+\mu_{l}+\varphi_{l}\right) E_{l} \\
& \frac{d I_{l}}{d t}=\rho_{l} E_{l}-\left(\mu_{l}+\sigma_{l}\right) I_{l} \\
& \frac{d R_{l}}{d t}=\varphi_{l} E_{l}-\left(\omega_{l}+\mu_{l}\right) R_{l}
\end{aligned}
$$

Livestock (1c)

The total populations for $N_{h}(t), N_{d}(t)$ and $N_{l}(t)$ are

$$
\begin{aligned}
& N_{h}(t)=S_{h}(t)+E_{h}(t)+I_{h}(t)+R_{h}(t) \\
& N_{d}(t)=S_{d}(t)+E_{d}(t)+I_{d}(t)+R_{d}(t) \\
& N_{l}(t)=S_{l}(t)+E_{l}(t)+I_{l}(t)+R_{l}(t)
\end{aligned}
$$

Therefore adding each of the differential equations of system (1) of human, dog and livestock population will give us.

$$
\begin{aligned}
& \frac{d N_{h}}{d t}=\vartheta_{h}-\mu_{h} N_{h}-\sigma_{h} I_{h} \\
& \frac{d N_{d}}{d t}=\vartheta_{d}-\mu_{d} N_{d}-\sigma_{d} I_{d} \\
& \frac{d N_{l}}{d t}=\vartheta_{l}-\mu_{l} N_{l}-\sigma_{l} I_{l}
\end{aligned}
$$

where $N_{i}, i=h, d, l$ is the total of human, dog and livestock population at time $\mathrm{t}$.

\section{Invariant Region}

The model system represented by (1) dealing with human, dog and livestock population will be analyzed in the feasible region, and all state variables and parameters are assumed to be positive for all $t \geq 0$. The invariant region will be obtained through Theorem 1 .

\section{Theorem 1:}

All solutions of the system (1) are contained in the region $\Phi \in \mathbb{R}_{+}^{12}$ and $\Phi=\Phi_{h} \cup \Phi_{d} \cup \Phi_{l} \in \mathbb{R}_{+}^{4} \times \mathbb{R}_{+}^{4} \times \mathbb{R}_{+}^{4}$.

Proof. The model system (1) was grouped in to Human $N_{h}$, Dog $N_{d}$ and livestock $N_{l}$. Such that

$$
\begin{aligned}
& \Phi_{h}=\left\{\left(S_{h}, E_{h}, I_{h}, R_{h}\right) \in \mathbb{R}_{+}^{4}: 0 \leq N_{h} \leq \frac{\vartheta_{h}}{\mu_{h}}\right\} \\
& \Phi_{d}=\left\{\left(S_{d}, E_{d}, I_{d}, R_{d}\right) \in \mathbb{R}_{+}^{4}: 0 \leq N_{d} \leq \frac{\vartheta_{d}}{\mu_{d}}\right\} \\
& \Phi_{l}=\left\{\left(S_{l}, E_{l}, I_{l}, R_{l}\right) \in \mathbb{R}_{+}^{4}: 0 \leq N_{l} \leq \frac{\vartheta_{l}}{\mu_{l}}\right\}
\end{aligned}
$$

and $\Phi$ is the positive invariant for system (1)

Thus $\Phi=\Phi_{h} \cup \Phi_{d} \cup \Phi_{l} \in \mathbb{R}_{+}^{4} \times \mathbb{R}_{+}^{4} \times \mathbb{R}_{+}^{4}$

From this it is sufficient to consider model system (1) in the region $\Phi$, and it can be shown to be positively invariant. The model can be considered as epidemiologically and mathematically well - posed.

\section{Model Analysis}

\subsection{Disease Free Equilibrium Points (DFE)}

To find the disease free equilibrium points we set the right hand side of equations of system (1) equal to zero. In the absence of attack or in the absence of rabies the following compartments will be zero.

$$
E_{h}=R_{h}=I_{h}=E_{d}=I_{d}=E_{l}=R_{l}=I_{l}=0
$$

then the disease free equilibrium (DFE) $\varepsilon_{0}$ will be

$$
\begin{aligned}
& \varepsilon_{0}=\left(S_{h}^{0}, 0,0,0, S_{d}, 0,0, R_{d}^{0}, S_{l}, 0,0,0\right) \\
& \text { where } S_{h}^{0}=\frac{\vartheta_{h}}{\mu_{h}}, \\
& S_{d}^{0}=\frac{\vartheta_{d}\left(\mu_{d}+\omega_{d}\right)}{\mu_{d}\left(\mu_{d}+\varphi_{d}+\omega_{d}\right)}, \\
& R_{d}^{0}=\frac{\varphi_{d} \vartheta_{d}}{\mu_{d}\left(\mu_{d}+\varphi_{d}+\omega_{d}\right)} \text { and } S_{h}^{0}=\frac{\vartheta_{l}}{\mu_{l}}
\end{aligned}
$$

For the dog population in the case of disease free equilibrium points $R_{d}$ cannot be zero because susceptible dogs which are vaccinated transfer to recovered class. Therefore the disease free equilibrium points of system (1) is exist and given by

$$
\begin{aligned}
& \varepsilon_{0}=\left(\frac{\vartheta_{h}}{\mu_{h}}, 0,0,0, \frac{\vartheta_{d}\left(\mu_{d}+\omega_{d}\right)}{\mu_{d}\left(\mu_{d}+\varphi_{d}+\omega_{d}\right)}, 0,0,\right. \\
& \left.\frac{\varphi_{d} \vartheta_{d}}{\mu_{d}\left(\mu_{d}+\varphi_{d}+\omega_{d}\right)}, \frac{\vartheta_{l}}{\mu_{l}}, 0,0,0\right)
\end{aligned}
$$

\subsection{The Basic Reproduction Number, $R_{0}$}

The basic reproduction number $R_{0}$ is a threshold parameter defined as the average number of secondary infection caused by an infectious individual by introducing in to a completely susceptible population. It is also called basic reproduction ratio or basic reproductive rate [5]. Basic reproduction number is very important parameter which helps to determine whether the disease spread in the population or it dies out. If $R_{0}<1$, then on average an infected individual produces less than one new infected individual over the course of its infectious period, and the infection cannot grow. Conversely, if $R_{0}>1$ then each infected individual produces, on average, more than one new infection, and the disease can invade the population. It is also crucial in the process of analyzing sensitive parameters which drive the dynamics of the disease and stability analysis 
of disease free and endemic equilibrium points.

To compute the basic reproduction number it is important to identify new infections from all other changes in the population. We used next generation operator method proposed by Van den Driessche \& Watmough [15]. We considered system (1) without vaccination i.e. $\omega=\varphi=0$.

Let $f_{i}(x)$ be the rate of appearance of new infection in compartment $i, v_{i}^{-}(x)$ be the rate of transfer of individuals out of compartment $i$ and $v_{i}^{+}(x)$ be the rate of transfer of individuals into compartment $i$ by all other means, and it is assumed that each function is continuously differentiable at least twice in each variable. The disease transmission model of system (1) consists of nonnegative initial conditions together with the following system of equations:

$$
\dot{x}=\mathcal{F}_{i}(x)=f_{i}(x)-v_{i}(x), \quad i=1, \ldots, 6
$$

where $v_{i}=v_{i}^{-}-v_{i}^{+}$

We consider expressions in which the infection is in progression. These are $E_{h}, I_{h}, E_{d}, I_{d}, E_{l}$ and $I_{l}$.

By rearranging equations of system (1) without vaccination from infected to infectious class of human dog and livestock population with a system of equations given by:

$$
\begin{aligned}
\frac{d E_{h}}{d t} & =\beta_{h} S_{h} I_{d}-\left(\rho_{h}+\mu_{h}\right) E_{h} \\
\frac{d I_{h}}{d t} & =\rho_{h} E_{h}-\left(\mu_{h}+\sigma_{h}\right) I_{h} \\
\frac{d E_{d}}{d t} & =\beta_{d} S_{d} I_{d}-\left(\rho_{d}+\mu_{d}\right) E_{d} \\
\frac{d I_{d}}{d t} & =\rho_{d} E_{d}-\left(\mu_{d}+\sigma_{d}\right) I_{d} \\
\frac{d E_{l}}{d t} & =\beta_{l} S_{l} I_{d}-\left(\rho_{l}+\mu_{l}\right) E_{l} \\
\frac{d I_{l}}{d t} & =\rho_{l} E_{l}-\left(\mu_{l}+\sigma_{l}\right) I_{l}
\end{aligned}
$$

Let $\mathrm{F}$ be a non - negative $n \times n$ matrix and $V$ be a non singular $\mathrm{N}$ - matrix such that

$$
\mathrm{F}=\left[\frac{\partial f_{i}\left(\varepsilon_{0}\right)}{x_{j}}\right] \text { and } \mathrm{V}=\left[\frac{\partial v_{i}\left(\varepsilon_{0}\right)}{x_{j}}\right] \text { with } 1 \leq i, j \leq 6
$$

The point $\varepsilon_{0}$ is the disease free equilibrium point in (2) without vaccination.

where

$$
f_{i}=\left[\begin{array}{l}
f_{1} \\
f_{2} \\
f_{3} \\
f_{4} \\
f_{5} \\
f_{6}
\end{array}\right]=\left[\begin{array}{l}
\beta_{h} S_{h} I_{d} \\
0 \\
\beta_{d} S_{d} I_{d} \\
0 \\
\beta_{l} S_{l} I_{d} \\
0
\end{array}\right]
$$

$$
v_{i}=\left[\begin{array}{l}
v_{1} \\
v_{2} \\
v_{3} \\
v_{4} \\
v_{5} \\
v_{6}
\end{array}\right]=\left[\begin{array}{l}
\left(\rho_{h}+\mu_{h}\right) E_{h} \\
\left(\mu_{h}+\sigma_{h}\right) I_{h}-\rho_{h} E_{h} \\
\left(\rho_{d}+\mu_{d}\right) E_{d} \\
\left(\mu_{d}+\sigma_{d}\right) I_{d}-\rho_{d} E_{d} \\
\left(\rho_{l}+\mu_{l}\right) E_{l} \\
\left(\mu_{l}+\sigma_{l}\right) I_{l}-\rho_{l} E_{l}
\end{array}\right]
$$

By considering the classes in which infection is on progression and using the linearization technique. The Jacobean matrices of $f$ and $v$ at the disease free equilibrium point $\varepsilon_{0}$ are given by:

$$
\begin{aligned}
\mathrm{F}=\frac{\partial f_{i}\left(\varepsilon_{0}\right)}{x_{j}}=\left[\begin{array}{cccccc}
0 & 0 & 0 & \frac{\beta_{h} \vartheta_{h}}{\mu_{h}} & 0 & 0 \\
0 & 0 & 0 & 0 & 0 & 0 \\
0 & 0 & 0 & \frac{\beta_{d} \vartheta_{d}}{\mu_{d}} & 0 & 0 \\
0 & 0 & 0 & 0 & 0 & 0 \\
0 & 0 & 0 & \frac{\beta_{l} \vartheta_{l}}{\mu_{l}} & 0 & 0 \\
0 & 0 & 0 & 0 & 0 & 0
\end{array}\right] \\
\mathrm{V}=\frac{\partial v_{i}\left(\varepsilon_{0}\right)}{\partial x_{j}}=
\end{aligned}
$$

$$
\left[\begin{array}{cccccc}
\rho_{h}+\mu_{h} & 0 & 0 & 0 & 0 & 0 \\
-\rho_{h} & \mu_{h}+\sigma_{h} & 0 & 0 & 0 & 0 \\
0 & 0 & \rho_{d}+\mu_{d} & 0 & 0 & 0 \\
0 & 0 & -\rho_{d} & \mu_{d}+\sigma_{d} & 0 & 0 \\
0 & 0 & 0 & 0 & \rho_{l}+\mu_{l} & 0 \\
0 & 0 & 0 & 0 & -\rho_{l} & \mu_{l}+\sigma_{l}
\end{array}\right]
$$

Solve for $V^{-1}$ and multiplying it with $F$ will give us a matrix $F V^{-1}=\left[\frac{\partial f_{i}\left(\varepsilon_{0}\right)}{x_{j}}\right]\left[\frac{\partial v_{i}\left(\varepsilon_{0}\right)}{x_{j}}\right]^{-1}$

Choosing the maximum eigenvalue in absolute terms that is the spectral radius of the matrix $F V^{-1}$ gives us the basic reproduction number which is given by:

$$
R_{0}=\frac{\rho_{d} \beta_{d} \vartheta_{d}}{\mu_{d}\left(\rho_{d}+\mu_{d}\right)\left(\mu_{d}+\sigma_{d}\right)}
$$

In our assumption there is no secondary infection in human and livestock population, all the parameters of $R_{0}$ in (9) are depending on dog population. This shows that targeting dog population in the process of combating rabies transmission is very important. Providing pre or post exposure prophylaxis for human or livestock population does not reduce the speared of the disease in the community. 


\subsection{The Effective Reproduction Number, $R_{e}$}

The effective reproduction number is defined as the measure of average number of infections caused by a single infectious individual introduced in a community in which intervention strategies (in our case vaccination) is administered. The effective reproduction number $R_{e}$ of system (1) is computed by applying the same procedure of $R_{0}$. The spectral radius (dominant eigenvalue) of $F V^{-1}$ denoted by $R_{e}=\rho\left(F V^{-}\right)$.

$$
R_{e}=\frac{\rho_{d} \beta_{d} \vartheta_{d}\left(\mu_{d}+\omega_{d}\right)}{\mu_{d}\left(\mu_{d}+\varphi_{d}+\omega_{d}\right)\left(\rho_{d}+\mu_{d}\right)\left(\mu_{d}+\sigma_{d}\right)}
$$

$$
\text { Since } R_{0}=\frac{\rho_{d} \beta_{d} \vartheta_{d}}{\mu_{d}\left(\rho_{d}+\mu_{d}\right)\left(\mu_{d}+\sigma_{d}\right)}
$$

we can express $R_{e}$ in terms of $R_{0}$ as

$$
R_{e}=\frac{\left(\mu_{d}+\omega_{d}\right)}{\left(\mu_{d}+\varphi_{d}+\omega_{d}\right)} R_{0}
$$

Numerical computation of. $R_{0}$, and $R_{e}$ was done using the data collected from Ethiopian Public Health Institute

Table 2. Parameter values.

\begin{tabular}{ll}
\hline Parameters & Value \\
\hline$\vartheta_{d}$ & $2 \times 10^{4}$ \\
$\sigma_{d}$ & 1 \\
$\omega_{d}$ & 0.5 \\
$\mu_{d}$ & 0.083 \\
$\beta_{d}$ & $1.29 \times 10^{-5}$ \\
$\rho_{d}$ & 0.17 \\
$\varphi_{d}$ & 0.1 \\
\hline
\end{tabular}

We substitute the parameter values to the expression found in (9) and (10)

$$
R_{0}=\frac{(0.17)\left(1.29 \times 10^{-5}\right)\left(2 \times 10^{4}\right)}{0.083(0.17+0.083)(0.083+1)} \approx 2
$$

Without any control measure the result of $R_{0}$ is greater than one, which shows that the disease will continue spreading in the population.

$$
R_{e}=\frac{(0.17)\left(1.29 \times 10^{-5}\right)\left(2 \times 10^{4}\right)(0.083+0.5)}{0.083(0.083+0.1+0.5)(0.17+0.083)(0.083+1)} \approx 1.6
$$

With the current $10 \%$ vaccination coverage for dog population the result of $R_{e}$ is greater than one which shows that the disease still persisting within and around Addis Ababa region.

The above results of $R_{e}$ and $R_{0}$ tell us that more control measures should be taken to control the spread of rabies within and around Addis Ababa.
We have simulated the effective reproduction number with different vaccination coverage and combination of vaccination, culling and controlling annual crop of newborn puppies. It shows that as we increase vaccination to the dog population there is a possibility of the disease dying out. The combination of vaccination, culling and controlling annual crop of newborn puppies are the best way to reduce $R_{e}$. On the simulation $R_{0}$ is without any vaccination, $R_{e 1}$ is the current $10 \%$ vaccination coverage, $R_{e 2}$ is $60 \%$ vaccination coverage. $R_{e 3}$ is the combination of $60 \%$ vaccination, $15 \%$ culling and declining $25 \%$ of annual crop of newborn puppies.

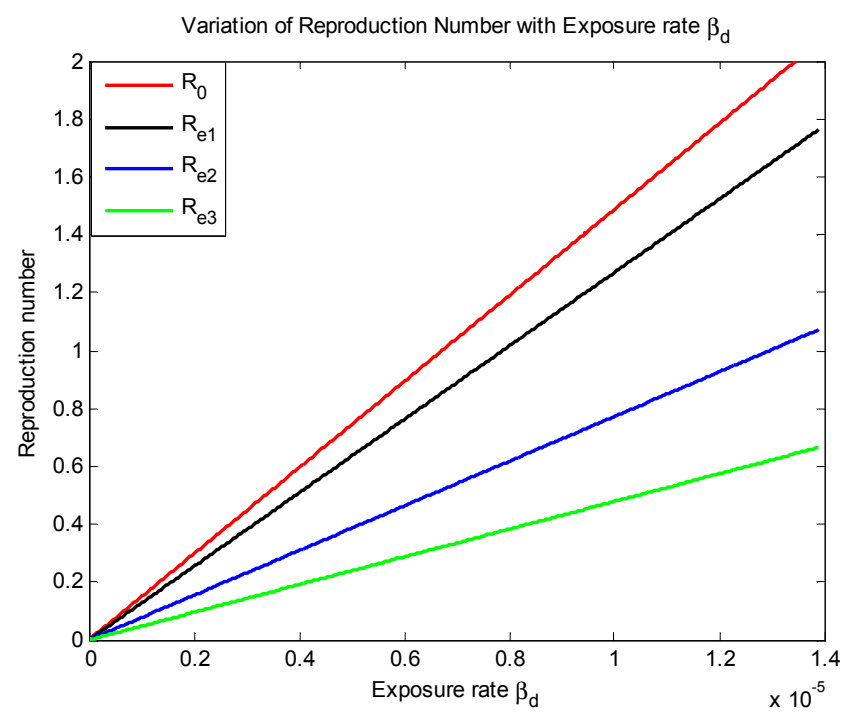

Figure 2. Reproduction number for different vaccination coverage and combination of vaccination, culling and controlling newborn puppies.

From Figure 2 we can observe that $R_{e 3}<R_{e 2}<R_{e 1}<R_{0}$, which shows that as we increase vaccination to $\operatorname{dog}$ population the effective reproduction number decreases and become less than one. Due to the high transmission rate of rabies from dogs to both human and livestock, we strongly recommend the combination of measures which makes the effective reproduction number less than one.

\section{Stability Analysis}

\subsection{Local Stability of the Disease Free Equilibrium Point}

In this sub - section we investigate the local stability of the disease free equilibrium point.

Theorem 1. If $R_{e}<1$, then (a) the disease - free equilibrium $\varepsilon_{0}$ of system (1) is locally asymptotically stable; (b) the disease - free equilibrium $\varepsilon_{0}$ of system (1) is globally asymptotically stable in the region $\Phi$.

From (2) the disease free equilibrium point is given by. 


$$
\begin{aligned}
& \varepsilon_{0}=\left(\frac{\vartheta_{h}}{\mu_{h}}, 0,0,0, \frac{\vartheta_{d}\left(\mu_{d}+\omega_{d}\right)}{\mu_{d}\left(\mu_{d}+\varphi_{d}+\omega_{d}\right)}, 0,0,\right. \\
& \left.\frac{\varphi_{d} \vartheta_{d}}{\mu_{d}\left(\mu_{d}+\varphi_{d}+\omega_{d}\right)}, \frac{\vartheta_{l}}{\mu_{l}}, 0,0,0\right)
\end{aligned}
$$

Next we derive the Jacobean matrix of system (1). This is done by differentiation of each of the equation of system (1) in terms of state variables

$$
S_{h}, E_{h}, I_{h}, R_{h}, S_{d}, E_{d}, I_{d}, R_{d}, S_{l}, E_{l}, I_{l}, R_{l}
$$

$J_{\varepsilon_{0}}=$

$\left[\begin{array}{ccccccccccccc}-\mu_{h} & 0 & 0 & \omega_{h} & 0 & 0 & \frac{-\beta_{h} \vartheta_{h}}{\mu_{h}} & 0 & 0 & 0 & 0 & 0 \\ 0 & -\left(\mu_{h}+\rho_{h}+\varphi_{h}\right) & 0 & 0 & 0 & 0 & \frac{\beta_{h} \vartheta_{h}}{\mu_{h}} & 0 & 0 & 0 & 0 & 0 \\ 0 & \rho_{h} & -\left(\mu_{h}+\sigma_{h}\right) & 0 & 0 & 0 & 0 & 0 & 0 & 0 & 0 & 0 \\ 0 & \varphi_{h} & 0 & -\left(\omega_{h}+\mu_{h}\right) & 0 & 0 & 0 & 0 & 0 & 0 & 0 & 0 \\ 0 & 0 & 0 & 0 & -\left(\mu_{d}+\varphi_{d}\right) & 0 & \frac{-\beta_{d} \vartheta_{d}\left(\mu_{d}+\omega_{d}\right)}{\mu_{d}\left(\mu_{d}+\varphi_{d}+\omega_{d}\right)} & \omega_{d} & 0 & 0 & 0 & 0 \\ 0 & 0 & 0 & 0 & 0 & -\left(\mu_{d}+\rho_{d}\right) & \frac{\beta_{d} \vartheta_{d}\left(\mu_{d}+\omega_{d}\right)}{\mu_{d}\left(\mu_{d}+\varphi_{d}+\omega_{d}\right)} & 0 & 0 & 0 & 0 & 0 \\ 0 & 0 & 0 & 0 & 0 & \rho_{d} & -\left(\mu_{d}+\sigma_{d}\right) & 0 & 0 & 0 & 0 & 0 \\ 0 & 0 & 0 & 0 & \varphi_{d} & 0 & 0 & -\left(\mu_{d}+\omega_{d}\right) & 0 & 0 & 0 & 0 \\ 0 & 0 & 0 & 0 & 0 & 0 & \frac{-\beta_{l} \vartheta_{l}}{\mu_{l}} & 0 & -\mu_{l} & 0 & 0 & 0 \\ 0 & 0 & 0 & 0 & 0 & 0 & \frac{\beta_{l} \vartheta_{l}}{\mu_{l}} & 0 & 0 & -\left(\mu_{l}+\rho_{l}+\varphi_{l}\right) & 0 \\ 0 & 0 & 0 & 0 & 0 & 0 & 0 & 0 & 0 & \rho_{l} & -\left(\mu_{l}+\sigma_{l}\right) & 0 \\ 0 & 0 & 0 & 0 & 0 & 0 & 0 & 0 & 0 & \varphi_{l} & 0 & -\left(\mu_{l}+\varphi_{l}\right)\end{array}\right]$

The trace of the above matrix will be

$$
\begin{gathered}
\operatorname{Tr}\left(J_{\varepsilon_{0}}\right)=-4 \mu_{d}-4 \mu_{h}-4 \mu_{l}-\rho_{d}-\rho_{h}-\rho_{l}-\sigma_{d}-\sigma_{h}-\sigma_{l}-\varphi_{d}-\varphi_{h}-2 \varphi_{l}-\omega_{d}-\omega_{h}<0 \\
\operatorname{Det}\left(J_{\varepsilon_{0}}\right)=\frac{\mu_{d} \mu_{h} \mu_{l}\left(-\beta_{d} \vartheta_{d}\left(\mu_{d}+\omega_{d}\right) \rho_{d}+\mu_{d}\left(\mu_{d}+\varphi_{d}+\omega_{d}\right)\left(\mu_{d}+\rho_{d}\right)\left(\mu_{d}+\sigma_{d}\right)\right)\left(\mu_{h}+\sigma_{h}\right)\left(\mu_{l}+\sigma_{l}\right)\left(\mu_{h}+\rho_{h}+\varphi_{h}\right)\left(\mu_{l}+\varphi_{l}\right)\left(\mu_{l}+\rho_{l}+\varphi_{l}\right)\left(\mu_{d}+\varphi_{d}+\omega_{d}\right)\left(\mu_{h}+\omega_{h}\right)}{\mu_{d}\left(\mu_{d}+\varphi_{d}+\omega_{d}\right)} \\
\text { Since } R_{e}=\frac{\rho_{d} \beta_{d} \vartheta_{d}\left(\mu_{d}+\omega_{d}\right)}{\mu_{d}\left(\mu_{d}+\varphi_{d}+\omega_{d}\right)\left(\rho_{d}+\mu_{d}\right)\left(\mu_{d}+\sigma_{d}\right)}
\end{gathered}
$$

$\operatorname{Det}\left(J_{\varepsilon_{0}}\right)=\mu_{d} \mu_{h} \mu_{l}\left(1-R_{e}\right)\left(\mu_{d}+\rho_{d}\right)\left(\mu_{d}+\sigma_{d}\right)\left(\mu_{h}+\sigma_{h}\right)\left(\mu_{l}+\sigma_{l}\right)\left(\mu_{h}+\rho_{h}+\varphi_{h}\right)\left(\mu_{l}+\varphi_{l}\right)\left(\mu_{l}+\rho_{l}+\varphi_{l}\right)\left(\mu_{d}+\rho_{d}+\varphi_{d}\right)\left(\mu_{h}+\omega_{h}\right)$

Thus for $R_{e}<1$, we have

$\operatorname{Tr}\left(J_{\varepsilon}\right)<0$ and $\operatorname{Det}\left(J_{\varepsilon_{0}}\right)>0$, then the DFE is locally asymptotically stable otherwise it is unstable if $R_{e}>1$.

\subsection{Global Stability of Disease Free Equilibrium \\ Points}

To investigate the global stability of disease free equilibrium point of system (1) we used the method proposed by [9].

We write our system as follows:

$$
\left\{\begin{array}{l}
\frac{d X_{n}}{d t}=A\left(X_{n}-X_{E_{0}, n}\right)+A_{1} X_{i} \\
\frac{d X_{i}}{d t}=A_{2} X_{i}
\end{array}\right.
$$

in which $X_{n}$ and $X_{i}$ are vectors corresponding to the transmitting and non - transmitting compartments, and $X_{E_{0}, n}$ is vector at disease free equilibrium point $E_{0}$ of the same vector length as $X_{n}$.

Referring to system (1) we define

$$
\begin{aligned}
& X_{n}=\left(S_{h}, R_{h}, S_{d}, R_{d}, S_{l}, R_{l}\right)^{T} \\
& X_{i}=\left(E_{h}, I_{h}, E_{d}, I_{d}, E_{l}, I_{l}\right)^{T}
\end{aligned}
$$

$$
X_{E_{0}, n}=\left(\frac{\vartheta_{h}}{\mu_{h}}, 0, \frac{\vartheta_{d}\left(\mu_{d}+\omega_{d}\right)}{\mu_{d}\left(\mu_{d}+\varphi_{d}+\omega_{d}\right)}, \frac{\varphi_{d} \vartheta_{d}}{\mu_{d}\left(\mu_{d}+\varphi_{d}+\omega_{d}\right)}, \frac{\vartheta_{l}}{\mu_{l}}, 0\right)^{T}
$$

$$
X_{n}-X_{E_{0}, n}=\left[\begin{array}{c}
S_{h}-\frac{\vartheta_{h}}{\mu_{h}} \\
R_{h} \\
S_{d}-\frac{\vartheta_{d}\left(\mu_{d}+\omega_{d}\right)}{\mu_{d}\left(\mu_{d}+\varphi_{d}+\omega_{d}\right)} \\
R_{d}-\frac{\varphi_{d} \vartheta_{d}}{\mu_{d}\left(\mu_{d}+\varphi_{d}+\omega_{d}\right)} \\
S_{l}-\frac{\vartheta_{h}}{\mu_{h}} \\
R_{l}
\end{array}\right]
$$


For the global stability of DFE we need to prove the following.

$A$ should be a matrix with real negative eigenvalues.

$A_{2}$ should be a Metzler matrix

Using system (1) together with the representation in (12) the two equations can be written as follows:

$$
\begin{gathered}
{\left[\begin{array}{l}
\vartheta_{h}+\omega_{h} R_{h}-\beta_{h} I_{d} S_{h}-\mu_{h} S_{h} \\
\varphi_{h} E_{h}-\left(\omega_{h}+\mu_{h}\right) R_{h} \\
\vartheta_{d}+\omega_{d} R_{d}-\left(\mu_{d}+\varphi_{d}+\beta_{d} I_{d}\right) S_{d} \\
\varphi_{d} S_{d}-\left(\mu_{d}+\omega_{d}\right) R_{d} \\
\vartheta_{l}+\omega_{l} R_{l}-\beta_{l} I_{d} S_{l}-\mu_{l} S_{l} \\
\varphi_{l} E_{l}-\left(\omega_{l}+\mu_{l}\right) R_{l}
\end{array}\right]=} \\
A\left[\begin{array}{l}
S_{h}-\frac{\vartheta_{h}}{\mu_{h}} \\
R_{h} \\
S_{d}-\frac{\vartheta_{d}\left(\mu_{d}+\omega_{d}\right)}{\mu_{d}\left(\mu_{d}+\varphi_{d}+\omega_{d}\right)} \\
R_{d}-\frac{\vartheta_{d} \varphi_{d}}{\mu_{d}\left(\mu_{d}+\varphi_{d}+\omega_{d}\right)} \\
S_{l}-\frac{\vartheta_{l}}{\mu_{l}} \\
R_{l}
\end{array}\right]+A_{1}\left[\begin{array}{l}
E_{h} \\
I_{h} \\
E_{d} \\
I_{d} \\
E_{l} \\
I_{l}
\end{array}\right]
\end{gathered}
$$

and

$$
\left[\begin{array}{l}
\beta_{d} S_{d} I_{d}-\left(\rho_{d}+\mu_{d}\right) E_{d} \\
\rho_{d} E_{d}-\left(\mu_{d}+\sigma_{d}\right) I_{d} \\
\beta_{d} S_{d} I_{d}-\left(\rho_{d}+\mu_{d}\right) E_{d} \\
\rho_{d} E_{d}-\left(\mu_{d}+\sigma_{d}\right) I_{d} \\
\beta_{l} I_{d} S_{l}-\left(\rho_{l}+\mu_{l}+\varphi_{l}\right) E_{l} \\
\rho_{l} E_{l}-\left(\mu_{l}+\sigma_{l}\right) I_{l}
\end{array}\right]=A_{2}\left[\begin{array}{l}
E_{h} \\
I_{h} \\
E_{d} \\
I_{d} \\
E_{l} \\
I_{l}
\end{array}\right]
$$

Matrices $A, A_{1}$ and $A_{2}$ are of order $6 \times 6$

Using non - transmitting elements of the Jacobian matrix of system (1) and representation in (12) we get.

$$
A=\left[\begin{array}{cccccc}
\mu_{d} & \omega_{h} & 0 & 0 & 0 & 0 \\
0 & -\left(\mu_{h}+\omega_{h}\right) & 0 & 0 & 0 & 0 \\
0 & 0 & -\left(\varphi_{d}+\mu_{d}\right) & \omega_{d} & 0 & 0 \\
0 & 0 & 0 & -\left(\mu_{d}+\omega_{d}\right) & 0 & 0 \\
0 & 0 & 0 & 0 & -\mu_{l} & \omega_{l} \\
0 & 0 & 0 & 0 & 0 & -\left(\omega_{l}+\mu_{l}\right)
\end{array}\right]
$$

$$
A_{1}=\left[\begin{array}{cccccc}
0 & 0 & 0 & -\beta S_{h} & 0 & 0 \\
\varphi_{h} & 0 & 0 & 0 & 0 & 0 \\
0 & 0 & 0 & -\beta_{d} S_{d} & 0 & 0 \\
0 & 0 & 0 & 0 & 0 & 0 \\
0 & 0 & 0 & -\beta_{l} S_{l} & 0 & 0 \\
0 & 0 & 0 & 0 & \varphi_{l} & 0
\end{array}\right]
$$

$$
A_{2}=\left[\begin{array}{cccccc}
-\left(\varphi_{h}+\mu_{h}+\rho_{h}\right) & 0 & 0 & \beta_{h} S_{h} & 0 & 0 \\
\rho_{h} & -\left(\mu_{h}+\sigma_{h}\right) & 0 & 0 & 0 & 0 \\
0 & 0 & -\left(\rho_{d}+\mu_{d}\right) & \beta_{d} S_{d} & 0 & 0 \\
0 & 0 & \rho_{d} & -\left(\mu_{d}+\sigma_{d}\right) & 0 & 0 \\
0 & 0 & 0 & \beta_{l} S_{l} & -\left(\rho_{l}+\mu_{l}+\varphi\right) & 0 \\
0 & 0 & 0 & 0 & \rho_{l} & -\left(\mu_{l}+\sigma_{l}\right)
\end{array}\right]
$$

We have seen that Matrix A is upper triangular whose eigenvalues are located on its main diagonal which is real and negative. $-\mu_{h},-\left(\mu_{h}+\omega_{h}\right),-\left(\varphi_{d}+\mu_{d}\right)$ $-\left(\mu_{d}+\omega_{d}\right),-\mu_{l},-\left(\omega_{l}+\mu_{l}\right)$. The off diagonal elements of matrix $A_{2}$ are non - negative (since all parameters are positive) which is Metzler matrix. This proves that the DFE point of system (1) globally asymptotically stable in the region $\Phi$. This leads us to the following important theorem.

Theorem 2 . The disease - free equilibrium point is globally asymptotically stable in the region $\Phi$ if $R_{e}<1$ and unstable if $R_{e}>1$.

\subsection{Endemic Equilibrium Points}

\subsubsection{Existence of Endemic Equilibrium Points}

To find the equilibrium points of system (1) we set the right hand side of the equation equal to zero. The endemic equilibrium points of system (1), when they exist are given by:

$$
\varepsilon_{0}^{*}=\left(S_{h}^{*}, E_{h}^{*}, I_{h}^{*}, R_{h}^{*}, S_{d}^{*}, E_{d}^{*}, I_{d}^{*}, R_{d}^{*}, S_{l}^{*}, E_{l}^{*}, I_{l}^{*}, R_{l}^{*}\right)
$$

Where

$$
\begin{aligned}
& S_{h}^{*}=\frac{\vartheta_{h}\left(\mu_{h}+\omega_{h}\right)+\left[\left(\omega_{h} \varphi_{h}-\left(\mu_{h}+\rho_{h}+\varphi_{h}\right)\left(\mu_{h}+\omega_{h}\right)\right]\right.}{\mu_{h}\left(\mu_{h}+\omega_{h}\right)} E_{h}^{*} \\
& E_{h}^{*}=\frac{\beta_{h} \vartheta_{h}\left(\mu_{h}+\omega_{h}\right) I_{d}^{*}}{\left(\mu_{h}+\omega_{h}\right)\left[\mu_{h}\left(\mu_{h}+\varphi_{h}+\rho_{h}\right)+\beta_{h} I_{d}^{*}\left(\mu_{h}+\varphi_{h}+\rho_{h}\right)\right]-\beta_{h} \omega_{h} \varphi_{h} I_{d}^{*}} \\
& I_{h}^{*}=\frac{\rho_{h} E_{h}}{\mu_{h}+\sigma_{h}} \\
& R_{h}^{*}=\frac{\varphi_{h} E_{h}^{*}}{\mu_{h}+\omega_{h}}
\end{aligned}
$$

$S_{d}^{*}=\frac{\left(\rho_{d}+\mu_{d}\right)\left(\sigma_{d}+\mu_{d}\right)}{\beta_{d} \rho_{d}} \quad E_{d}^{*}=\frac{\left(\sigma_{d}+\mu_{d}\right)}{\rho_{d}} I_{d}^{*}$

$\left.I_{d}^{*}=\frac{\vartheta_{d}-\mu_{d} N_{d}^{*}}{\sigma_{d}} \quad R_{d}^{*}=\frac{\varphi_{d}\left(\rho_{d}+\mu_{d}\right)\left(\sigma_{d}+\mu_{d}\right)}{\beta_{d} \rho_{d}\left(\mu_{d}+\omega_{d}\right)}\right\}$

$S_{l}^{*}=\frac{\vartheta_{l}\left(\mu_{l}+\omega_{l}\right)+\left[\left(\omega_{l} \varphi_{l}-\left(\mu_{l}+\rho_{l}+\varphi_{l}\right)\left(\mu_{l}+\omega_{l}\right)\right]\right.}{\mu_{l}\left(\mu_{l}+\omega_{l}\right)} E_{l}^{*}$

$E_{l}^{*}=\frac{\beta_{l} \vartheta_{l}\left(\mu_{l}+\omega_{l}\right) I_{d}^{*}}{\left(\mu_{l}+\omega_{l}\right)\left[\mu_{l}\left(\mu_{l}+\varphi_{l}+\rho_{l}\right)+\beta_{l} I_{d}^{*}\left(\mu_{l}+\varphi_{l}+\rho_{l}\right)\right]-\beta_{l} \omega_{l} \varphi_{l} I_{d}^{*}}$

$$
I_{l}^{*}=\frac{\rho_{l} E_{l}}{\mu_{l}+\sigma_{l}} \quad R_{l}^{*}=\frac{\varphi_{l} E_{l}^{*}}{\mu_{l}+\omega_{l}}
$$

in which

$$
I_{d}^{*}=\frac{\vartheta_{d} \beta_{d} \rho_{d}\left(\mu_{d}+\omega_{d}\right)+\mu_{d}\left(\mu_{d}+\rho_{d}\right)\left(\mu_{d}+\sigma_{d}\right)\left(\mu_{d}+\varphi_{d}+\omega_{d}\right)}{\beta_{d}\left(\mu_{d}+\omega_{d}\right)\left(\mu_{d}+\rho_{d}\right)\left(\mu_{d}+\sigma_{d}\right)}
$$




\subsubsection{Local Stability of the Endemic Equilibrium}

To show the local stability of the endemic equilibrium points of system (1) we have used the following theorem as in [13].

Theorem 3 (Routh - Hurwitz Criteria) given a polynomial

$$
p(\lambda)=\lambda^{n}+a_{1} \lambda^{n-1}+\ldots+a_{n-1} \lambda+a_{n},
$$

Where the coefficients $a_{i}$ are real constants, $\mathrm{i}=1, \ldots \mathrm{n}$, define the n Hurwitz matrices using the coefficients $a_{i}$ of the characteristic polynomial:

$$
H_{1}=\left(a_{1}\right), H_{2}=\left(\begin{array}{cc}
a_{1} & 1 \\
a_{3} & a_{2}
\end{array}\right), \quad H_{3}=\left(\begin{array}{ccc}
a_{1} & 1 & 0 \\
a_{3} & a_{2} & a_{1} \\
a_{5} & a_{4} & a_{3}
\end{array}\right)
$$

and $H_{n}=\left(\begin{array}{cccccc}a_{1} & 1 & 0 & 0 & \cdots & 0 \\ a_{3} & a_{2} & a_{1} & 1 & \cdots & 0 \\ a_{5} & a_{4} & a_{3} & a_{2} & \cdots & 0 \\ \vdots & \vdots & \vdots & \vdots & \cdots & \vdots \\ 0 & 0 & 0 & 0 & \cdots & a_{n}\end{array}\right)$

where $a_{i}=0$ if $j>n$. All of the roots of the polynomial $p(\lambda)$ are negative or have negative real part iff the determinants of all Hurwitz matrices are positive:

$$
\operatorname{det} H_{j}>0, \quad j=0,1,2, \ldots, n \text {. }
$$

Details on Routh - Hurwitz criteria are given by [2, 13]

Now consider system (1a) as it is independent of (1b) and (1c). The Jacobian matrix of system (1a) is given by:

$$
\left.J\right|_{\varepsilon_{*}}=\left[\begin{array}{cccc}
-\mu_{h}-\beta_{h} I_{d}^{*} & 0 & 0 & \omega_{h} \\
\beta_{h} I_{d}^{*} & -\mu_{h}-\varphi_{h}-\rho_{h} & 0 & 0 \\
0 & \rho_{h} & -\mu_{h}-\sigma_{h} & 0 \\
0 & \varphi_{h} & 0 & -\mu_{h}-\omega_{h}
\end{array}\right]
$$

Through computation, we derive the characteristic polynomial

$$
\begin{aligned}
p(\lambda)= & \lambda^{4}+(a+b+c+d) \lambda^{3}+ \\
& (a b+a c+a d+b c+b d+c d) \lambda^{2} \\
& +\left(a b c+a b d+a c d+b c d-\beta_{h} I_{d}^{*} \varphi_{h} \omega_{h}\right) \lambda \\
& +a b c d-\beta_{h} I_{d}^{*} \varphi_{h} \omega_{h}
\end{aligned}
$$

where $a=\mu_{h}+\beta_{h} I_{d}^{*}, \quad b=\mu_{h}+\rho_{h}+\varphi_{h}$,

$$
c=\mu_{h}+\sigma_{h}, d=\mu_{h}+\omega_{h}
$$

the expressions for $I_{d}^{*}$ and $\mathrm{S}_{d}^{*}$ are as in (16).

we now consider

$$
p(\lambda)=\lambda^{4}+A \lambda^{3}+B \lambda^{2}+C \lambda+D
$$

$$
\begin{gathered}
A=a+b+c+d \\
B=a b+a c+a d+b c+b d+c d \\
C=a b c+a b d+a c d+b c d-\beta_{h} I_{d}^{*} \varphi_{h} \omega_{h} \\
D=a b c d-\beta_{h} I_{d}^{*} \varphi_{h} \omega_{h}
\end{gathered}
$$

Through calculation we have come up with the following characteristic polynomial.

$$
\begin{aligned}
& p_{1}(\lambda)=\lambda^{4}+\left(a_{1}+b_{1}+c_{1}+d_{1}\right)+\left(a_{1} b_{1}+a_{1} c_{1}+a_{1} d_{1}+b_{1} c_{1}+b_{1} d_{1}+\right. \\
& \left.c_{1} d_{1}-\rho_{1} \beta_{1} S_{d}^{*}-\varphi_{d} \omega_{d}\right) \lambda^{2}+\left(a_{1} c_{1} b_{1}+a_{1} b_{1} d_{1}+a_{1} c_{1} d_{1}+b_{1} c_{1} d_{1}+\right. \\
& \left.\beta_{d}^{2} I_{d}^{*} \rho_{d} S_{d}^{*}-\varphi_{d} \omega_{d} b_{1}-\varphi_{d} \omega_{d} c_{1}-a_{1} \rho_{d} \beta_{d} S_{d}^{*}-d_{1} \rho_{d} \beta_{d} S_{d}^{*}\right) \lambda+ \\
& \left(a_{1} b_{1} c_{1} d_{1}+\varphi_{d} \rho_{d} \omega_{d} \beta_{d} S_{d}^{*}+\beta_{d}^{2} I_{d}^{*} \rho_{d} S_{d}^{*}-a_{1} d_{1} \rho_{d} \beta_{d} S_{d}^{*}-\varphi_{d} \omega_{d} c_{1} b_{1}\right)
\end{aligned}
$$

Using the characteristic polynomial representation in (18) the Hurwitz matrix is given by

$$
H_{4}=\left(\begin{array}{llll}
A & 1 & 0 & 0 \\
C & B & A & 1 \\
0 & D & C & B \\
0 & 0 & 0 & D
\end{array}\right)
$$

The determinant of the Hurwitz matrix is

$$
-D\left(A^{2} D-A B C+C^{2}\right)
$$

By Routh - Hurtwiz criteria of Theorem 3 the determinant of Hurtwiz matrix becomes positive if the following conditions hold true.

$$
A>0, \quad C>0, \quad D>0 \text { and } A B C>C^{2}+A^{2} D
$$

All parameters of our model are positive and the starred variables $I_{d}^{*}$ and $\mathrm{S}_{d}^{*}$ are given by (16)

Therefore $A=a+b+c+d$ is greater than zero, $C>0$ if and only if $(a b c+a b d+a c d+b c d)>\beta_{h} I_{d}^{*} \varphi_{h} \omega_{h}, D>0$ if and only if $a b c d>\beta_{h} I_{d}^{*} \varphi_{h} \omega_{h}$.When all the conditions for $A, C$ and $D$ hold, similarly using the same procedure $A B C>C^{2}+A^{2} D$ holds. Hence all roots of the characteristic polynomial of (18) are negative, this verify that system (1a) is locally asymptotically stable.

Further we consider system (1b)

The Jacobean is given by

$\left.J\right|_{\mathcal{E}_{*}}=\left[\begin{array}{cccc}-\left(\mu_{d}+\varphi_{d}+\beta_{d} I_{d}^{*}\right) & 0 & -\beta_{d} S_{d}^{*} & \omega_{d} \\ \beta_{d} I_{d}^{*} & -\left(\mu_{d}+\rho_{d}\right) & \beta_{d} S_{d}^{*} & 0 \\ 0 & \rho_{d} & -\left(\mu_{d}+\sigma_{d}\right) & 0 \\ \varphi_{d} & 0 & 0 & -\left(\mu_{d}+\omega_{d}\right)\end{array}\right]$

where

Where 


$$
\begin{aligned}
& a_{1}=\mu_{d}+\varphi_{d}+\beta_{d} I_{d}^{*} \\
& b_{1}=\mu_{d}+\rho_{d} \\
& c_{1}=\mu_{d}+\sigma_{d} \text { and } d_{1}=\mu_{d}+\omega_{d}
\end{aligned}
$$

and the expressions for $I_{d}^{*}$ and $\mathrm{S}_{d}^{*}$ are as in (16).

Consider

$$
p_{1}(\lambda)=\lambda^{4}+A_{1} \lambda^{3}+B_{1} \lambda^{2}+C_{1} \lambda+D_{1}
$$

where

$$
\begin{aligned}
A_{1}= & a_{1}+b_{1}+c_{1}+d_{1} \\
B_{1}= & a_{1} b_{1}+a_{1} c_{1}+a_{1} d_{1}+b_{1} c_{1}+b_{1} d_{1}+c_{1} d_{1}- \\
& \rho_{d} \beta_{d} S_{d}^{*}-\varphi_{d} \omega_{d} \\
C_{1}= & a_{1} c_{1} b_{1}+a_{1} b_{1} d_{1}+a_{1} c_{1} d_{1}+b_{1} c_{1} d_{1}+\beta_{d}^{2} I_{d}^{*} \rho_{d} S_{d}^{*} \\
& -\varphi_{d} \omega_{d} b_{1}-\varphi_{d} \omega_{d} c_{1}-a_{1} \rho_{d} \beta_{d} S_{d}^{*}-d_{1} \rho_{d} \beta_{d} S_{d}^{*}, \\
D_{1}= & a_{1} b_{1} c_{1} d_{1}+\varphi_{d} \rho_{d} \omega_{d} \beta_{d} S_{d}+\beta_{d}^{2} I_{d}^{*} \rho_{d} S_{d}^{*}- \\
& a_{1} d_{1} \rho_{d} \beta_{d} S_{d}^{*}-\varphi_{d} \omega_{d} c_{1} b_{1}
\end{aligned}
$$

Using the characteristic polynomial representation in (22) the Hurwitz matrix is given by:

$$
H_{4}^{\prime}=\left(\begin{array}{cccc}
A_{1} & 1 & 0 & 0 \\
C_{1} & B_{1} & A_{1} & 1 \\
0 & D_{1} & C_{1} & B_{1} \\
0 & 0 & 0 & D_{1}
\end{array}\right)
$$

It follows that the determinant of $H_{4}^{\prime}$ is

$$
-D_{1}\left(A_{1}^{2} D_{1}-A_{1} B_{1} C_{1}+C_{1}^{2}\right)
$$

By Routh - Hurtwiz criteria of theorem 3 the determinant of Hurtwiz matrix becomes positive if the following conditions hold.

$$
\begin{aligned}
& A_{1}>0, \quad C_{1}>0, \quad D_{1}>0 \quad \text { and } \\
& A_{1} B_{1} C_{1}>C_{1}^{2}+A_{1}^{2} D_{1}
\end{aligned}
$$

Since all the parameters of our model are positive and the variables $I_{d}^{*}$ and $\mathrm{S}_{d}^{*}$ are given by (16)

$A_{1}=a_{1}+b_{1}+c_{1}+d_{1}$ is greater than zero, $C_{1}>0$ if and only if $\left(a_{1} c_{1} b_{1}+a_{1} b_{1} d_{1}+a_{1} c_{1} d_{1}+b_{1} c_{1} d_{1}+\beta_{d}^{2} I_{d}^{*} \rho_{d} S_{d}^{*}\right)>$

$$
\left(\varphi_{d} \omega_{d} b_{1}+\varphi_{d} \omega_{d} c_{1}+a_{1} \rho_{d} \beta_{d} S_{d}^{*}+d_{1} \rho_{d} \beta_{d} S_{d}^{*}\right)
$$

and $D_{1}>0$ if and only if.

$$
\begin{gathered}
\left(a_{1} b_{1} c_{1} d_{1}+\varphi_{d} \rho_{d} \omega_{d} \beta_{d} S_{d}+\beta_{d}^{2} I_{d}^{*} \rho_{d} S_{d}^{*}\right)> \\
\left(a_{1} d_{1} \rho_{d} \beta_{d} S_{d}^{*}+\varphi_{d} \omega_{d} c_{1} b_{1}\right)
\end{gathered}
$$

When all the conditions for $A_{1}, C_{1}$ and $D_{1}$ hold, similarly using the same procedure $A_{1} B_{1} C_{1}>C_{1}^{2}+A_{1}^{2} D_{1}$ holds. Hence all roots of the characteristic polynomial of (22) are negative, This verify that system (1b) is locally asymptotically stable

By using the same procedure for (1c) will give us the same proof. Therefore the endemic equilibrium point of system (1) is locally asymptotically stable.

\section{Conclusion}

In this paper we have formulated and analyzed a deterministic mathematical model for the dynamics of rabies transmission. The model comprises dog, human and livestock population. Since classical rabies is very common in dog population, the model was intended to show rabies transmission among dogs and to human and livestock. The dog population infects both human and livestock.

The basic reproduction number and the effective reproduction number have been computed using next generation operator. The results are depending only on the parameter of dog population. The reason behind is that in our assumption there is no secondary infection in human and livestock or among themselves. This tells us that, control measures should focus on dog population. Supplying post exposure prophylaxis for human can save exposed individuals, but cannot reduce the future rabies transmission. For a specified set of values of parameters as deduced from the data provided by Ethiopian Public Health Institute of Addis Ababa, the basic reproduction number $R_{0}$ and the effective reproductive number $R_{e}$ works out to be 2 and 1.6, which indicates the disease will be endemic

The numerical simulation for the reproduction ratio shows that the combination of $60 \%$ vaccination, $15 \%$ culling of stray dogs and $25 \%$ reduction of annual crop of new born puppies are the best measure to control rabies transmission within and around Addis Ababa.

We have computed the disease free and endemic equilibrium points. If the effective reproduction number $R_{e}<1$ the disease free equilibrium is globally asymptotically stable and the disease dies out. The endemic equilibrium points have been driven whenever they exist. Using the Routh - Hurwitz Criteria, the endemic equilibrium points are locally asymptotically stable.

\section{Acknowledgments}

The authors would like to thank ACBF of The Nelson Mandela African Institution of Science and Technology (NM - AIST) for the sponsorship.

\section{References}

[1] K. M. Addo, An SEIR Mathematical Model for Dog Rabies. Case Study: Bongo District, Ghana, MSc. Dissertation Kwame Nkrumah University of Science and Technology, 2012.

[2] S. N. Sivanandam and S. N. Deepa, Linear system design using Routh Column Polynomials. Songklanakarin J. Sci. Technol. 2007, 29 (6): 1651 - 1659. 
[3] A. Ali, F. Mengistu, K. Hussen, G. Getahun, A. Deressa, E. Yimer and K. Tafese, Overview of Rabies in and around Addis Ababa, in Animals Examined in EHNRI Zoonoses Laboratory Between, 2003 and 2009, Ethiopian Veterinary Journal, 14 (2010), 91 - 101.

[4] C. Castillo - Chavez, Z. Feng, and W. Huang, Mathematical Approaches for Emerging and Re - emerging Infectious Diseases, An Introduction. Springer Verlag, (2002).

[5] N. Chitnis, J. Hyman, J. Cushing, Determining important parameters in the spread of malaria through the sensitivity analysis of a malaria model, Bull. Math. Biology. 70 (2008), 1272 - 1296.

[6] A. Deressa, A. Ali, M. Bayene, N. Selassie, E. Yimer and K. Hussen, The status of rabies in Ethiopia: A retrospective record review, Ethiopian Journal of Health Development. 24 (2010), 1 - 6 .

[7] H. Hethcote, The mathematics of infectious diseases SIAM Review. 42 (2000), 599 - 653.

[8] Q. Hou, Z. Jin and S. Ruan, Dynamics of rabies epidemics and the impact of control efforts in Guangdong Province, China, Journal of theoretical biology, 300 (2012)39 - 47.

[9] A. Iggidr, J. Mbang, G. Sallet\& J. J. Tewa. Multi compartment models, Discrete and Continuous Dynamical Systems series S, 2007 (Special), 506 - 519.

[10] W. T. Jemberu, W. Molla, G. Almaw and S. Alemu, Incidence of rabies in humans anddomestic animals and people's awareness in North Gondar Zone, Ethiopia, PLoS Negl. Trop. Dis. 7 (2013).

[11] S. Khan, Rabies molecular virology, diagnosis, prevention and treatment, 2012. Available oline at http: //www. biomedcentral. com/ content/ pdf/1743 - 422X - 9 - 50. pdf. Retrieved 13 April 2015.

[12] A. L. Lloyd and S. Valeika, Network models in epidemiology: an overview, Complex population dynamics: nonlinear modeling in ecology, epidemiology and genetics, 2007. Available online at http: //infoserve. sandia.gov/sand_doc/ 2008 /086044.pdf. Retrieved 13 April 2015

[13] P. C. Parks, A new proof of the Routh - Hurwitz stability criterion using the second method of Liapunov. In Mathematical Proceedings of the Cambridge Philosophical Society, Cambridge University Press, 58 (1962), 694 - 702.

[14] T. Reta, S. Teshale, A. Deresa, A. Ali, F. Mengistu, D. Sifer and C. Freuling, Rabies in animals and humans in and around Addis Ababa, the capital city of Ethiopia: A retrospective and questionnaire based study, Journal of Veterinary Medicine and Animal Health, 6 (2014), 178 - 186.

[15] P. Van der Driessche and J. Watmough, Reproduction numbers and sub threshold endemic equilibria for compartmental models of disease transmission, Math. Biosciences. 180 (2002), 29 - 48.

[16] World Health Organization, WHO Expert Consultation on Rabies. Second report, World Health Organization technical report series, p. 1, 2013.

[17] M. Yousaf, M. Qasim, S. Zia, R. Khan, U. Ashfaq and S. Khan, Rabies molecular virology, diagnosis, prevention and treatment, Virology Journal, 9 (2012), 1 - 5.

[18] J. Zhang, Z. Jin, G. Q. Sun, T. Zhou and S. Ruan, Analysis of rabies in China: transmission dynamics and control, PLoS One, 6 (2011), p. e20891. 